\title{
Neutrino Dispersion Relations from a Dark Energy Model
}

\author{
Emilio Ciuffoli, ${ }^{1}$ Jarah Evslin, ${ }^{1}$ Jie Liu, ${ }^{2}$ and Xinmin Zhang $^{1,2}$ \\ ${ }^{1}$ TPCSF, IHEP, Chinese Academy of Sciences, Yu Quan Lu 19(B), Beijing 100049, China \\ ${ }^{2}$ Theoretical Physics Division, IHEP, Chinese Academy of Sciences, Yu Quan Lu 19(B), Beijing 100049, China \\ Correspondence should be addressed to Jarah Evslin; jarah@ihep.ac.cn
}

Received 23 October 2012; Accepted 7 November 2012

Academic Editors: K. Cho, F.-H. Liu, and A. Romanino

Copyright (C) 2013 Emilio Ciuffoli et al. This is an open access article distributed under the Creative Commons Attribution License, which permits unrestricted use, distribution, and reproduction in any medium, provided the original work is properly cited.

We consider a model in which the neutrino is kinetically coupled to a scalar field and study its implications for environmentally dependent neutrino velocities. Following the usual effective field theory logic, this coupling is expected to arise in neutrino dark energy models. It leads to a Lorentz violation in the neutrino sector. The coupling of the scalar field to the stress tensor of the Earth yields terrestrial neutrino dispersion relations distinct from those in interstellar space.

\section{Introduction}

Last year the OPERA experiment announced the arrival of 16,111 Swiss neutrinos, 61 nanoseconds ahead of schedule [1]. It turns out that this announcement was incorrect [2, 3] and in the past year nearly all of the long baseline neutrino experiments [4-8] including OPERA itself [1] have confirmed that neutrinos travel at the speed of light to within experimental errors. But just as the velocity anomaly has disappeared, other anomalies and inconsistencies in the standard model with two or three mass neutrinos have been reinforced.

The velocity anomaly at OPERA immediately was observed to be in contradiction with the measurement of the velocities of the neutrinos from SN1987A $[9,10]$. There were attempts to evade this inconsistency with artificial models of energy dependences [11] but these were found to be in contradiction with simple kinematic arguments $[12,13]$. The only models which could not be falsified on theoretical grounds alone were those which radially changed the nature of spacetime $[14,15]$ and those which introduced an environmental dependence in the neutrino mass [16], although the later was found to be inconsistent with fifth force constraints [17], which was remedied in [18, 19]. In fact environmentally dependent neutrino models have been used to explain anomalies while avoiding various constraints for almost a decade $[20,21]$.
Similarly there appears to be room to resolve today's anomalies using environmental dependence. For example, the LSND experiment [22] and MiniBooNE [23-25, 25, 26] now both report an anomalous electron (anti)neutrino surplus which excludes the standard 3-neutrino oscillation paradigm at $3.8 \sigma$. Nearly all of their parameter space is ruled out by the fact that no deficit was observed at KARMEN [27] or ICARUS [28]. While the KARMEN and LSND experiments are nearly identical, the KARMEN baseline has a much lower average density than the LSND baseline. In addition, inside of a gallium detector both the GALLEX [29, 30 ] and SAGE experiments [31-34] report a $2.5 \sigma$ deficit of neutrinos from a radioactive source. In addition, tens of short baseline [35] and $1 \mathrm{~km}$ baseline [36] reactor antineutrino experiments have found about a $6 \%$ flux deficit. Finally, at even higher densities, the expected upturn in solar neutrinos at low density has not been observed and there may even be evidence for a downturn $[37,38]$.

One explanation for these anomalies could be sterile neutrinos; however, combining ICARUS, KARMEN, LSND, and MiniBooNE constraints one finds a sterile neutrino mass of about $0.5 \mathrm{eV}$ [28] while the gallium anomaly requires a mass about $0.8 \mathrm{eV}$ or else there would be no oscillation inside of the 2 meter detectors and the solar anomaly requires a mass of a millielectron volt or less. Three flavors of sterile neutrinos are strongly excluded by cosmological constraints. While constraints arising from primordial helium abundance in HII 
regions are fraught with systematic errors and controversy, the last years South Pole Telescope's measurement of the $\mathrm{CMB}$ diffusion radius [39] alone together with standard big bang nucleosynthesis and WMAP7's measurement of the sound horizon [40] provide a reasonably definitive exclusion of 3 sterile neutrino models. Within the next year the new South Pole Telescope and Planck data will provide the last word on the number of flavors of light particles which were once in thermal equilibrium with the primordial plasma, providing a firm upper bound on the number of sterile neutrinos which are sufficiently strongly coupled to explain the anomalies listed in the previous paragraph.

Perhaps the correct explanation for these anomalies, like the neutrino velocity anomaly reported by OPERA, is experimental error. However, it is difficult to ignore the fact that they all occur in dense environments, such as the Earth, the iron shielding of LSND, gallium detectors, and even the core of the sun. The lowest density environment in which an anomaly has been reported is ice, where a horizontal neutrino excess has been reported by AMANDA and confirmed by IceCube [41]. However, even in that case, this is a relative excess and could easily be instead a deficit of neutrinos which pass through the Earth at steeper angles. The deficit saturates around 10-15 degrees, corresponding to a maximum baseline in the Earth and so could, for example, result from oscillation to a sterile neutrino which may or may not be more massive inside of the Earth.

Summarizing, it remains a logical possibility that these anomalies are caused by some beyond the standard model matter effects. After all, neutrinos are the only particles which can travel more than $1 \mathrm{~km}$ through solid rock, and so they are the only particles which may be sensitive to such new physics.

This motivates a systematic study of effective field theories encoding potential environmental effects on neutrinos. While a large body of literature exists on beyond the standard model interactions of conventional matter on neutrinos, in the present work we will attempt a systematic study of a different class of models, which were first presented as explanations for the superluminality that had been claimed by OPERA. These are models in which neutrinos interact not with the usual standard model particles, but with a dark coherent scalar field which is coupled gravitationally to other matter. Such a dark scalar, if it has a fixed profile and fills a small portion of the universe, so that it does not increase in volume as it expands, will in recent times contribute to the density and pressure of the universe with a nonrelativistic equation of state and so will be a source of dark matter. If on the other hand it nearly homogeneously fills most of the universe during some period, then it will contribute to the density and pressure with an equation of state $w=-1$ and so contribute to the dark energy during that period. For simplicity and for a connection with the literature on this subject we will simply refer to the scalar field as the dark energy field, regardless of whether it ever had an equation of state close to -1 . In this note we will not attempt to use these models to solve the anomalies described above.

\section{Neutrino Coupling and Dispersion Relations}

The models which we will consider invariably modify the neutrino dispersion relations. This change necessarily violates Lorentz symmetry. We will consider spontaneous violations of Lorentz symmetry, which arise by adding terms to the Lagrangian which couple a new field to neutrino bilinears. The couplings and the new field are similar to those introduced in the neutrino dark energy model of [20], and to a large extent to those in the earlier models of $[42,43]$, although we do not demand that the additional field actually provides the observed dark energy. The field acquires a VEV due to interactions with the Earth, which spontaneously breaks the Lorentz symmetry. In $[16,44,45]$ such models were constructed in which the new fields introduced were, respectively, a symmetric tensor, a vector, and a scalar. We will consider a scalar field $\Pi$, which as explained below will have the advantage that it requires the tuning of only a single parameter. The fact that our couplings resemble those which arise in the neutrino dark energy scenario of [20] yields a cosmological justification for the exclusiveness of these terms to the neutrino sector.

In an effective field theory setting, it is sufficient to consider the operators of lowest dimension which preserve Lorentz invariance. The terms with no derivatives can be absorbed into redefinitions of the fields and parameters of the effective theory. Strong upper bounds on these terms arise, for example, on masses from beta decay and as a result these terms will be negligible at OPERA energies.

As the standard model Higgs field has not yet been discovered. (A new boson has been discovered, but the $6 \sigma$ confidence of the discovery arises from a $2 \gamma$ excess which is greater than that of the standard model Higgs and so cannot be considered to be evidence for the standard model Higgs. The standard model Higgs does not yet fit the data $5 \sigma$ better than the standard model with no Higgs, although it may before the scheduled shut down.) For the sake of generality we will not yet restrict our attention to a particular mechanism of SU(2) gauge symmetry breaking. We will write the effective theory directly in terms of the neutrino fields, allowing the gauge symmetry to be realized either linearly or nonlinearly. Later, in (6) we will specialize to the standard GWS symmetry breaking and we will see that this choice changes the naive dimension of our new operator, as factors of the Higgs field need to be added. While the absolute dimension is indeed shifted, the relative dimensions of the different possible operators are not altered and therefore our interaction continues to be the dominant relevant interaction in the infrared.

We will be interested only in terms which cannot be reabsorbed into other terms via field definitions up to terms without derivatives. In general the modifications of the dispersion relation can be linear or quadratic in the new couplings; we will eventually restrict our restriction to the linear modifications and so to the coupling terms which lead to linear modifications. With all of these criteria, we are left with

$$
\Delta \mathscr{L}=\frac{1}{2}\left(i a_{\mu} \bar{v} \partial^{\mu} v+i c_{\mu \nu} \bar{\gamma} \gamma^{\mu} \partial^{v} v-d_{\mu \nu \rho} \bar{v} \gamma^{\mu} \partial^{v} \partial^{\rho} v\right),
$$


where $a, c$, and $d$ are tensors constructed from derivatives of $\Pi$. In our dimensional analysis scheme, in which the coefficients are constructed from a scalar field of dimension $[m], d$ in the last term is of higher dimensionality and so need not be considered, but we will keep it during this section for illustration as a linear energy dependence of neutrino superluminality is excluded at OPERA by less than two sigmas.

Clearly these derivative terms are nontrivial only if $\Pi$ is not constant. The anomalies discussed above have been seen within the Earth and Sun, while SN1987A neutrinos tightly constrain the dispersion relation in space; therefore, these derivatives need to be localized on Earth. The simplest possibility would be if $\dot{\Pi}$ was localized on Earth and vanishes in space, while $\partial_{k} \Pi$ vanishes everywhere. However, it is easy to see that if $\Pi$ begins with a constant value, the spatial gradients between here and SN1987A will dominate over the temporal gradient in less than a mere 160,000 years, much less than the age of the Earth. Therefore such field configurations are not logically consistent.

The next simplest possibility is that $\dot{\Pi}$ is negligible but the gradient of $\Pi$ lies along the Earth's radial direction $r$. In Section 3 we will describe a prototypical example of a scalar field with the desired behavior. For now, it will simply be relevant that the only nonzero derivatives, and so the only nonzero components of the tensors $a, c$, and $d$, will be those with only $r$ indices or an even number of identical tangential indices. Then the dispersion relation is

$$
E=\sqrt{P^{\prime 2}+M^{\prime 2}}
$$

with $i$ th spatial component

$$
P_{i}^{\prime}=p_{i}+c_{i j} p_{j}+d_{i j k} p_{j} p_{k}, \quad M^{\prime}=m+a_{j} p_{j},
$$

where sums over repeated indices are understood.

Notice that as in the model of [44] and unlike that of [16] the dispersion relation is anisotropic. In particular, the velocity of a neutrino is

$$
v_{i}=\frac{\partial E}{\partial p_{i}}=\frac{a_{i} M^{\prime}+\left(\delta_{i j}+c_{i j}+2 d_{i j k} p_{k}\right) P_{j}^{\prime}}{E} .
$$

Let $x$ be the radial coordinate and choose $y$ direction to be orthogonal to $x$ such that the velocity of a given neutrino is in the $x-y$ plane, at an angle $\theta$ with respect to $x$. Considering only the leading contributions to the Lorentz-violating terms, this neutrino will travel with a fractional superluminality equal to

$$
\begin{aligned}
\frac{v-c}{c} \simeq & \frac{a_{x}^{2}}{2} \cos ^{2} \theta+\left(c_{x x} \cos ^{2} \theta+c_{y y} \sin ^{2} \theta\right) \\
& +2 E \cos \theta\left(d_{x x x} \cos ^{2} \theta+3 d_{x y y} \sin ^{2} \theta\right) .
\end{aligned}
$$

We recall that, since the tensors $a, c$, and $d$ are proportional to the derivative of the scalar field $\Pi$, coefficients without an even number of identical tangential indices (such as $c_{x y}$ and $\left.d_{y y y}\right)$ are zero.
As mentioned above in the sequel we will ignore the last term as it is created by a higher dimensional operator in our effective field theory description. Notice that $c_{x x}$ and $a_{x}$ only occur in the combination $c_{x x}+a_{x}^{2} / 2$ at this precision. The terms which we have omitted are strongly suppressed, by factors of the Lorentz-violating coefficients (which we assume to be at least of the same order of magnitude as the superluminality fraction) or even by the ratio of the neutrino rest mass to its energy, and so the identification of further terms or even the separate identification of $c_{x x}$ and $a_{x}$ will need to await a much more precise or qualitatively different experimental setting. As we can then not hope to experimentally distinguish between the effects of $c_{x x}$ and $a_{x}$, we will simply neglect $a_{x}$ in what follows. Working with the effective lagrangian with a $S U_{L}(2) \times U_{Y}(1)$ symmetry, the second operator in (1) is given by

$$
\Delta \mathscr{L}=-b\left(\partial^{\mu} \partial^{v} \Pi\right)\left(H^{\dagger} \bar{L}\right) \gamma_{\mu} \partial_{v}(L H),
$$

where we have defined the constant $b$ :

$$
c_{\mu \nu}=-\frac{b\langle v\rangle^{2}}{2}\left\langle\partial_{\mu} \partial_{v} \pi\right\rangle,
$$

and the $H$ is the Higgs doublet of the standard model. In [45] the author considers a term which couples the neutrino kinetic tensor to $\partial_{\mu} \Pi \partial_{v} \Pi$. However, this term is of one energy dimension greater than (6) and so is suppressed according to the usual logic of effective field theories.

\section{The Model}

For concreteness we will consider models in which the neutrino propagator is modified by a kinetic coupling of the neutrino to a field which obtains a VEV, if this messenger field is coupled to the Earth. In [46], for example, the authors proposed that this spatial dependence can emerge in type IIB string theory model.

No such coincidence is required in models in which the messenger is coupled directly to baryon density or to the background stress tensor in such a way that it acquires a classical expectation value concentrated near massive objects. So long as this classical field drops off sufficiently quickly from the sources, it will affect terrestrial neutrinos and not appreciably affect supernova neutrinos. However, it is important that the field drops off sufficiently quickly so that the Earth's effects dominate preferably over those of the Sun and certainly over those of the center of the Galaxy. In [16] this was achieved by adding spin two fields whose inverse mass is fixed by hand to be roughly the inverse radius of the Earth (and necessarily less than the inverse distance to the Sun), while the coupling was chosen to yield the OPERA superluminality. In this paper we will present an alternative model in which the effects of the Sun and Galaxy are suppressed not by tuning another parameter, but simply by the derivative structure of our coupling to the neutrinos.

We consider a model with the neutrino dark energy term (6) coupling the neutrino to a scalar field with or without 
a minimal Galileon coupling [47] given by the boundary DGP model [48]:

$$
\mathscr{L}_{\Pi}=-\frac{1}{2} \partial_{\mu} \Pi \partial^{\mu} \Pi-\frac{a}{2}(\square \Pi)\left(\partial_{v} \Pi\right)^{2}+4 \sqrt{3 \pi G_{N}} \Pi T .
$$

The coefficient $a$, which can be taken to be zero, is a parameter of dimension $\left[l^{3}\right]$ which parameterizes the nonlinear Galileon interaction. $T$ is the trace of the stress tensor of all of the matter, except for the scalar. The coupling of $\Pi$ to the stress tensor could in principle lead to fifth forces beyond experimental bounds, as described in a very similar setting in [17], however, a quick calculation shows that only the product of the coefficient of this coupling and the coefficient $b$ in (6) appears in the neutrino $v-c$; therefore any reduction of the coefficient of the stress tensor coupling which may be mandated by fifth force constraints can be compensated by an opposite rescaling of $b$. While the Lagrangian itself has higher derivative terms, terms in the equations of motion have at most two derivatives acting on each $\Pi$, which allows the existence of ghost-free solutions such as that which we will use.

The Galileon interaction term is useful because it reduces short distance singularities, via the Vainshtein mechanism [49], at least in the presence of spherically symmetric stress tensor sources. More precisely, for an external source of mass $M$ there will be a distance scale [48]:

$$
R=\frac{\left(\pi G_{N}\right)^{1 / 6}}{\sqrt{3}}(4 a M)^{1 / 3}
$$

at which the behavior of the $\Pi$ field changes. We can choose the Galileon coupling $a$ such that this distance is either larger than or smaller than the radius of the Earth $r$. For concreteness, in the rest of this paper, we will chose $a$ to be sufficiently small so that $R \ll r$, and so we will set $a=0$ in the rest of this section. A small value of $a$ is useful for, among other things, avoiding the potential formation of closed timelike curves [50]. However, $a$ will again become important for distance scales smaller than $R$; for example, it may be invoked for neutrino phenomenology in neutron stars and, depending on its value, in the cores of massive stars.

Let us now calculate a static, spherically symmetric field configuration $\Pi(r)$ in the presence of a nonrelativistic matter source with density $\rho(r)$. The $\Pi$ equation of motion is then

$$
-4 \sqrt{3 \pi G_{N}} \rho=\nabla^{2} \Pi=\frac{1}{r^{2}} \partial_{r}\left(r^{2} \partial_{r} \Pi\right) .
$$

This is easily integrated to yield

$$
r^{2} \partial_{r} \Pi=-4 \sqrt{3 \pi G_{N}} \int_{\tilde{r}=0}^{\tilde{r}=r} \widetilde{r}^{2} \rho d \tilde{r}=-\sqrt{\frac{3 G_{N}}{\pi}} M(r),
$$

where $M(r)$ is the density contained in the object up to a radius $r$, and we have fixed the constant of integration by imposing that $\Pi$ is differentiable at the origin.
One can now easily find the second derivatives of $\Pi$, which appear in the coupling (6). Choosing our coordinates such that $y=z=0$ at a given point,

$$
\begin{gathered}
\partial_{z}^{2} \Pi=\partial_{y}^{2} \Pi=\frac{1}{r} \partial_{r} \Pi=-\sqrt{\frac{3 G_{N}}{\pi}} \frac{M(r)}{r^{3}}, \\
\partial_{x}^{2} \Pi=\nabla^{2} \Pi-\partial_{y}^{2} \Pi-\partial_{z}^{2} \Pi=-4 \sqrt{3 \pi G_{N}}\left(\rho(r)-\frac{M(r)}{2 \pi r^{3}}\right)
\end{gathered}
$$

while terms with mixed derivatives vanish. In particular, in the Earth's crust

$$
\begin{aligned}
& \partial_{x}^{2} \Pi=4 \sqrt{3 \pi G_{N}}\left(\frac{2}{3} \rho_{0}-\rho\right), \\
& \partial_{y}^{2} \Pi=\partial_{z}^{2} \Pi=-4 \sqrt{\frac{\pi G_{N}}{3}} \rho_{0},
\end{aligned}
$$

where $\rho$ and $\rho_{0}$ are, respectively, the average densities of the crust and of the Earth as a whole. More generally, (14) may be applied to a point at any radius $r$ in a background with an arbitrary spherically symmetric density profile if one identifies $\rho$ with the density at radius $r$ and $\rho_{0}$ with the average density at radii less than $r$.

\section{Fitting}

4.1. SN1987A. The equation (6) modifies the dispersion relation for the neutrinos, for example, allowing their velocity to deviate from the usual relativistic form. In the language of [16] this corresponds to a modification of the effective metric in which the neutrinos propagate. The neutrino velocity at an angle $\theta$ with respect to the radial direction is given by inserting (7) into (5):

$$
\epsilon=\frac{v-c}{c}=-\frac{b\langle v\rangle^{2}}{2}\left(\partial_{x}^{2} \Pi(r) \cos ^{2}(\theta)+\partial_{y}^{2} \Pi(r) \sin ^{2}(\theta)\right),
$$

where $\langle v\rangle$ is the Higgs VEV. In the case of even the longest baseline neutrino experiments to date, $\cos ^{2}(\theta) \sim .01$ and so we will ignore the first term. Using (14) one finds

$$
\epsilon=\frac{v-c}{c}=2 b \sqrt{\frac{\pi G_{N}}{3}}\langle v\rangle^{2} \rho_{0} \sim(800 \mathrm{eV})^{5} b,
$$

where we have used $\rho_{0} \sim 5.5 \mathrm{gm} / \mathrm{cm}^{3}$. For example, the OPERA result $\epsilon \sim 3 \times 10^{-5}$ would be reproduced if

$$
b \sim \frac{1}{(6 \mathrm{keV})^{5}} .
$$

This is much smaller than the energies of the OPERA neutrinos, which means that this effective field theory approach is invalid for deviations from relativistic dispersion relations as large as those reported by OPERA. The effective field theory approach can only be trusted below the cutoff energy.

Of course, this fit is only reasonable if the main source of the dark energy field is the Earth. In other words, the main 
contribution to $\Pi$ must arise from its coupling to the Earth, and not, for example, the Sun or the matter in our Galaxy. In the case of a distance object, the $\rho$ term in (13) vanishes, while the other terms are simply the ratio of the total mass to the distance cubed. Therefore, at the surface of the Earth, the contribution of the Sun (with respect to that of the Earth) is suppressed by a factor of $10^{-8}$ and that of the Galaxy by $10^{-25}$; thus, it is reasonable to ignore these contributions. In the case of the model in [16] the corresponding $M / R$ dependence would lead to a dominant contribution from the Sun and Galaxy, and so these contributions are eliminated by hand by choosing the mass of their new field to be of order of the inverse radius of the Earth.

And so what about neutrinos from SN1987A? The fractional superluminality for these neutrinos needs to be less than $10^{-9}$. This is easily satisfied, as one can see by directly calculating the change in arrival time, or else simply noting that nearly all of the trip is in interstellar space, where $\epsilon$ is of order $10^{-30}$. In fact, examining (13) more closely, one sees that during the first part of their trip the neutrinos are actually subluminal. The subliminality constraints on SN1987A neutrinos are very weak, as they depend upon assumptions about the propagation of light in the various media between here and the large Magellanic cloud.

4.2. KamLAND. KamLAND lies between 55 nuclear reactors, which supply it with antineutrinos. The electron neutrinos oscillate into muon neutrinos. If the energy difference between an electron and muon neutrino is $\Delta E$, the probability that a given electron neutrino is still an electron neutrino after traveling a distance $L$ is

$$
1-\sin ^{2}\left(2 \theta_{e \mu}\right) \sin ^{2}\left(\frac{L \Delta E}{2}\right),
$$

where $\theta_{e \mu}$ is the mixing angle between these two flavors. Using the dispersion relation (2) one readily finds, for an energy $E$ neutrino, that the latter phase is

$$
\begin{aligned}
& L\left(\frac{\delta m^{2}}{2 E}+\left(a_{z}^{(e) 2}+2 c_{z z}^{(e)}-a_{z}^{(\mu) 2}-2 c_{z z}^{(\mu)}\right) E \cos ^{2} \theta\right. \\
& \quad+2\left(c_{y y}^{(e)}-c_{y y}^{(\mu)}\right) E \sin ^{2} \theta+\left(2\left(d_{x x x}^{(e)}-d_{x x x}^{(\mu)}\right) \cos ^{2} \theta\right) \\
& \left.\quad+3\left(d_{x y y}^{(e)}-d_{x y y}^{(\mu)}\right) \sin ^{2} \theta+3\left(d_{x y y}^{(e)}-d_{x y y}^{(\mu)}\right) E^{2} \cos ^{2} \theta\right)
\end{aligned}
$$

where $\delta m^{2}$ is the difference between the squared masses of the two relevant neutrino mass eigenstates and the $e$ and $\mu$ superscripts denote the Lorentz-violating neutrino couplings to the two flavors, respectively.

This can be easily applied to models that had been proposed for the neutrino velocity anomaly. Inserting coefficients $2 c+a^{2}$ which are sufficiently large to fit the OPERA data, together with an energy $E=5 \mathrm{MeV}$ typical of reactor neutrinos seen above the background at KamLAND, one finds that neutrinos oscillate about every 5 nanometers. This is much smaller than the resolution at KamLAND, and so KamLAND would in this case find an energy-independent survival probability. This is in grave contradiction with KamLAND's results in [51] which present two full averaged neutrino oscillations. Higher order terms, such as the $d$ term, lead to an even shorter wavelength but with an $E$ dependence which cannot cancel that of previous terms. Therefore, a cancellation in such terms, in order to be consistent with KamLAND's results, must be imposed order by order in $E$, and in fact also order in order in $\cos (\theta)$, which is small but still larger than the ratio of the energy to the neutrino mass. Such a cancellation is not possible with the terms in the lowest orders of the effective action considered in this note.

4.3. Solar Neutrinos and the MSW Effect. Our model modifies the neutrino dispersion relations not only near the Earth, but near any massive body. In fact the effect is even more pronounced near the Sun, although unlike the model of [16] the modification of the fractional neutrino velocity in the core of the Sun is still quite small. This modification means that experimental signatures for our model may already be apparent in the solar neutrino data, and in particular solar neutrino data provides a nontrivial experimental check on the viability of our proposal. The velocity of solar neutrinos cannot be determined directly, as one does not know the time at which they were emitted. However, the interaction of neutrinos with the Sun leads to a further modification of their dispersion relations which has an observed effect on neutrino oscillation, called the MSW effect [52-54]. This leads one to ask whether the modification to the neutrino dispersion relation which we propose modifies the experimental signatures of the MSW effect, in particular whether it modifies the energy dependence of the electron neutrino survival probability.

In this subsection we will modify a textbook [55] derivation of the solar electron neutrino survival probability, applying the superluminal dispersion relation of our model. We will calculate the change in the survival probability for neutrinos of low enough energy such that their flavors are converted adiabatically, as is the case for observed solar neutrinos. While we will find that this effect is much too small to be observed for solar neutrinos, we will note that it is potentially large enough to be observed some day for supernova neutrinos.

The relevant electroweak interaction of neutrinos with leptons and baryons can be described by the Lagrangian density term of the form

$$
\Delta \mathscr{L}=\bar{v} A \gamma_{0} P_{L} v
$$

If we consider only two flavors (as is reasonable for rough calculations involving solar neutrinos) we can express the interaction parameter $A$ as

$$
A=\sqrt{2} G_{F}\left[\frac{N_{e}}{2}\left(\begin{array}{cc}
1 & 0 \\
0 & -1
\end{array}\right)-\frac{N_{n}-N_{e}}{2}\left(\begin{array}{ll}
1 & 0 \\
0 & 1
\end{array}\right)\right],
$$

where $N_{e}$ and $N_{n}$ are the electron and neutron densities, respectively. We consider, for simplicity, neutrinos which travel radially outwards from the center of the Sun, in the 
direction which we will call $\widehat{x}$. Recall from (14) that the main contribution to the superluminality fraction $\epsilon$ arises from

$$
c_{x x}^{\odot}=-2 b\left\langle v^{2}\right\rangle \sqrt{3 \pi G_{N}}\left(\frac{2}{3} \rho_{0}^{\odot}-\rho^{\odot}\right),
$$

where $c_{x \mathrm{x}}^{\odot}$ is value of the Lorentz-violating (LV) coefficient defined in (7) inside of the Sun. $\rho^{\odot}(r)$ and $\rho_{0}^{\odot}(r)$ are the solar density at radius $r$ and the mean density at radius less than $r$, respectively. The $r$ dependence will usually be left implicit.

In the case of the OPERA experiment, since the neutrino velocity is nearly perpendicular to the radial direction, the main contribution to the superluminality was proportional to the value of the LV coefficient inside of the Earth $c_{y y}^{\oplus}=$ $2 b\left\langle v^{2}\right\rangle \sqrt{\pi G_{N}} \rho_{0}^{\oplus} / \sqrt{3}$.

In the center of the Sun, $\rho_{0}^{\odot}(0)=\rho^{\odot}(0)$, and hence $c_{x x}^{\odot}$ and $c_{y y}^{\odot}$ are equal:

$$
\begin{aligned}
\left(\frac{2}{3} \rho_{0}(0)-\rho(0)\right) & =-\frac{1}{3} \rho_{C}^{\odot} \Longrightarrow c_{x x}^{\odot}(0) \\
& =2 b\left\langle v^{2}\right\rangle \sqrt{\frac{\pi G_{N}}{3}} \rho_{C}^{\odot} \\
& =\frac{\rho_{C}^{\odot}}{\rho_{0}^{\oplus}} \epsilon^{\oplus} \simeq 8 \cdot 10^{-4},
\end{aligned}
$$

where $\epsilon^{\oplus}$ is the superluminality inside of the Earth's crust (for tangential motion), $\rho_{C}^{\odot}$ is the density in the center of the Sun, and $\rho_{0}^{\oplus}$ is the average density of the Earth.

In order to calculate $\rho_{0}^{\odot}$ at a generic point, we need the approximate density profile of the solar core:

$$
\rho^{\odot}(r) \simeq \rho_{C}^{\odot} \exp \left[-\frac{10 r}{R_{\odot}}\right] \text {. }
$$

On the boundary of solar core $\left(r=0.2 R_{\odot}\right)$,

$$
\begin{gathered}
\left(\frac{2}{3} \rho_{0}\left(0.2 R_{\odot}\right)-\rho\left(0.2 R_{\odot}\right)\right) \simeq \frac{1}{40} \rho_{C}^{\odot} \\
\Longrightarrow c_{x x}^{\odot}\left(0.2 R_{\odot}\right)=-\frac{3 \rho_{C}^{\odot}}{40 \rho_{0}^{\oplus}} \epsilon^{\oplus} \\
\simeq-0.6 \cdot 10^{-4} .
\end{gathered}
$$

This means that at the center of the Sun the neutrinos are superluminal but at higher radii they slow and by the boundary of the core they are subluminal. Crucially, at every radius the dimensionless $\mathrm{LV}$ parameter $c_{x x}^{\odot}$ is much smaller than one, and so we can use it to perturbatively expand all quantities of interest.

Including the interaction term $A$, in the ultrarelativistic limit $E \gg m$ our dispersion relation (2) becomes

$$
E_{\mathrm{LV}} \simeq\left(1+c_{x x}^{\odot}\right) p+\left(\frac{m \cdot m^{\dagger}}{2 E_{\mathrm{LV}}}+A\right),
$$

where we have defined the mass matrix

$$
m \cdot m^{\dagger}=V\left(\begin{array}{cc}
m_{1}^{2} & 0 \\
0 & m_{2}^{2}
\end{array}\right) V^{T}, \quad V=\left(\begin{array}{cc}
\cos \theta & \sin \theta \\
-\sin \theta & \cos \theta
\end{array}\right) .
$$

The subscript LV indicates that the dispersion relation is Lorentz violating. The evolution of a neutrino planewave wavefunction in the electroweak interaction basis is given by multiplication by

$$
e^{-i p x+i E_{\mathrm{LV}} t}=e^{\left(-i p v+i E_{\mathrm{LV}}\right) t}=e^{\left(-i p\left(1+c_{x x}^{\odot}\right)+i E_{\mathrm{LV}}\right) t}=e^{i H t}
$$

where we have defined the matrix $H$, which can be evaluated using (26):

$$
H=\left(\frac{m \cdot m^{\dagger}}{2 E_{\mathrm{LV}}}\right)+A .
$$

The matrix $H$ has the same form as in the Lorentzinvariant $(\mathrm{LI})$ case, but now $E_{\mathrm{LV}} \simeq\left(1+c_{x x}^{\odot}\right) p$ instead of $E \simeq p$.

In our two-flavor approximation, the mixing angle and the difference between the two eigenvalues can be found diagonalizing matrix $H$ :

$$
\begin{aligned}
H= & \frac{m_{1}^{2}+m_{2}^{2}+2 \sqrt{2} G_{F} E_{\mathrm{LV}}\left(N_{e}-N_{n}\right)}{4 E_{\mathrm{LV}}}\left(\begin{array}{ll}
1 & 0 \\
0 & 1
\end{array}\right) \\
& +\frac{\Delta m^{2}}{4 E_{\mathrm{LV}}}\left(\begin{array}{cc}
-\cos 2 \theta+\delta_{\mathrm{LV}} & \sin 2 \theta \\
-\sin 2 \theta & \cos 2 \theta-\delta_{\mathrm{LV}}
\end{array}\right),
\end{aligned}
$$

where

$$
\delta_{\mathrm{LV}}=\frac{2 \sqrt{2} G_{F} E_{\mathrm{LV}} N_{e}}{\Delta m^{2}} \simeq 7.6 \cdot 10^{-8} \frac{E_{\mathrm{LV}} / \mathrm{MeV}}{\Delta m^{2} / \mathrm{eV}^{2}} \frac{\rho}{\mathrm{g} / \mathrm{cm}^{3}} Y_{e},
$$

and $Y_{e}=Z / A$ is the electron fraction. The terms proportional to the identity matrix affect neither the difference $\Delta m_{m}^{2}$ between the eigenvalues of $H$ nor the rotation angle $2 \theta_{m}$ which diagonalizes it

$$
\begin{gathered}
\left.\Delta m_{m}^{2}\right|_{\mathrm{LV}}=\Delta m^{2} \sqrt{\left(\cos (2 \theta)-\delta_{\mathrm{LV}}\right)^{2}+\sin ^{2}(2 \theta)}, \\
\left.\sin \left(2 \theta_{m}\right)^{2}\right|_{\mathrm{LV}}=\frac{\sin (2 \theta)}{\sqrt{\left(\cos (2 \theta)-\delta_{\mathrm{LV}}\right)^{2}-\sin ^{2}(2 \theta)}} .
\end{gathered}
$$

Expanding in $c_{x x}^{\odot},(32)$ yield

$$
\begin{gathered}
\left.\Delta m_{m}^{2}\right|_{\mathrm{LV}} \simeq \Delta m_{m}^{2}\left(1-\frac{\delta(\cos (2 \theta)-\delta)}{\lambda} c_{x x}^{\odot}\right), \\
\left.\sin ^{2}\left(2 \theta_{m}\right)\right|_{\mathrm{LV}} \simeq \sin ^{2}\left(2 \theta_{m}\right)\left(1+\frac{\delta(\cos (2 \theta)-\delta)}{\lambda} c_{x x}^{\odot}\right),
\end{gathered}
$$

where

$$
\lambda=(\cos 2 \theta-\delta)^{2}+\sin ^{2} 2 \theta
$$

and $\delta$ is obtained from $\delta_{\mathrm{LV}}$ by replacing the Lorentz-violating dispersion relation with the usual one:

$$
\delta=\frac{2 \sqrt{2} G_{F} E N_{e}}{\Delta m^{2}} .
$$


is

A typical value of the LV coefficient $c_{x x}$ inside of the core

$$
\frac{1}{0.2 R_{\odot}} \int_{0}^{0.2 R_{\odot}} c_{x x}(r) d r \simeq-\frac{1}{4} \frac{\rho_{C}^{\odot}}{\rho^{\oplus}} \epsilon^{\oplus} \simeq-2 \cdot 10^{-4} .
$$

Taking $\Delta m^{2} \simeq 10^{-5} \mathrm{eV}^{2}, \sin (2 \theta) \simeq 0.9$, using the typical values for the solar core $\rho^{\odot} \simeq 80 \mathrm{~g} / \mathrm{cm}^{3}, Y_{e} \simeq 1$, and $|p| \simeq 1 \mathrm{MeV}$, the fractional difference between the Lorentzviolating and the Lorentz-invariant effective mass squared difference is

$$
\frac{\left.\Delta m_{m}^{2}\right|_{\mathrm{LV}}-\Delta m_{m}^{2}}{\Delta m_{m}^{2}} \simeq-\frac{\left.\sin \left(2 \theta_{m}\right)^{2}\right|_{\mathrm{LV}}-\sin \left(2 \theta_{m}\right)^{2}}{\sin \left(2 \theta_{m}\right)^{2}} \simeq 4 \cdot 10^{-6},
$$

which is much smaller than the fractional uncertainties in the values of $\Delta m^{2}$ and $\sin \left(2 \theta_{m}\right)^{2}$.

Moreover, since the solar radius is much larger than the oscillation length, the surviving probability of an electron neutrino detected on the Earth is (neglecting the effect of the matter in the Earth)

$$
P_{\left(v_{e} \rightarrow v_{e}\right)}=\frac{1}{2}+\frac{1}{2} \cos (2 \theta) \cos \left(2 \theta_{m}\right) .
$$

In our LV case we find

$$
\begin{aligned}
& \left.P_{\left(v_{e} \rightarrow v_{e}\right)}\right|_{\mathrm{LV}} \\
& =P_{\left(v_{e} \rightarrow v_{e}\right)}\left(1-\frac{(\cos (2 \theta)-\delta) \cos (2 \theta) \sin ^{2}(2 \theta) \delta}{\lambda\left(\sqrt{\lambda-\sin ^{2}(2 \theta)} \sqrt{\lambda}+\cos (2 \theta) \sqrt{\lambda-\sin ^{2}(2 \theta)}\right)} c_{x x}\right) \\
& \simeq P_{\left(v_{e} \rightarrow v_{e}\right)}\left(1-0.1 \cdot c_{x x}\right) .
\end{aligned}
$$

In the case of solar neutrinos $c_{x x}=c_{x x}^{\odot} \sim 10^{-4}$ and so we obtain a correction to the neutrino survival probability of order $10^{-5}$, which is well within the experimental errors of solar neutrino observations and also within the uncertainties within which the relevant parameters are known. Thus, no deviation with respect to solar neutrino experiments is expected.

If, on the other hand, the MSW effect is observed for supernova neutrinos then the adiabatic conversion assumed here is unreliable. However, the high density involved leads to a $c_{x x}$ of at least order unity, and so the corresponding correction to the conversion rate in this model will be appreciable, perhaps allowing for the exclusion of the model.

\section{Experimental Signatures}

Once one has included the interaction term (6) to explain superluminal neutrino velocities here on Earth, one needs to deal with its consequences throughout our universe. In particular, it may become appreciable inside of various astrophysical objects. In the case of a spherically symmetric object, (14) tells us that the superluminality at a radius $r$ only depends on the density $\rho$ at that radius and the average density at lower radii $\rho_{0}$. This may seem peculiar, since there is no Gauss' law for $\Pi$. Indeed, any choice of boundary conditions for $\Pi$ implies that its value in the core of an object depends on the $\rho$ profile at higher radii. However, in our model, and not in that of $[16,44]$, the superluminality only depends on the second derivatives of $\Pi$, which is independent of the constants of integration and so of these boundary conditions. This freedom in choosing boundary conditions is necessary for many cosmological applications of the $\Pi$ field, such as the dark energy model of [20] and Galileon cosmologies.

In the core of a spherically symmetric object, in which the density is approximately constant, the deviation from the relativistic dispersion relations is easily approximated. Up to geometrical factors whose magnitude and sign depend on the direction in which the neutrino travels, the fractional superluminosity of neutrinos is simply given by the ratio of the deviation on Earth to the ratio of the average density of the Earth to that of the object's core. For example, in the case of the core of our Sun, whose density varies between 4 and 30 times that of the Earth, the OPERA velocity anomaly would predict a neutrino fractional subluminosity or superluminosity of order between $10^{-4}$ and $10^{-3}$. This change in the neutrino's dispersion relation affects its propagator and so may in principle have observable consequences on the various fusion processes occurring in our solar core, perhaps allowing for an exclusion or verification of our model. This is in contrast with the model of [16], in which neutrino superluminality is of order 1 at the surface of the Sun and persists at greater depths including the outer core, where a significant amount of fusion occurs.

However, even in our model the superluminality consistency with OPERA's claim would have led to order 1 deviations from relativity whenever the density reaches about $2 \times 10^{5} \mathrm{~g} / \mathrm{cm}^{3}$. This level is easily surpassed, for example, in the cores of older massive stars which fuse carbon or heavier elements [56] and is even reached in white dwarves. Thus in these situations one may expect drastic departures from the standard Lorentz-invariant quantum field theory predictions for amplitudes and decay rates involving the neutrino propagator, potentially affecting, for example, models of the helium flash. This fact also implies that such models are in general incompatible with anomalies as large as those reported by OPERA.

Another crucial distinction between our model and that of [16] is that our neutrino velocity is direction dependent; radially traveling neutrinos on the surface of the Earth are subluminal. For shallow angles such as those of current accelerator neutrino experiments, the deviation from the relativistic dispersion relations in our model is reasonably independent of the angle, there is only a correction of order of the angle $(\pi / 2-\theta)$ squared. On the other hand, in the second model of [44], the superluminality is proportional to the cosine of the angle with respect to a preferred direction, which given the symmetries of the problem will likely correspond to the Earth's radial direction.

\section{Electron Superluminality}

In addition to the experimental constraints described above one may add that bounds on terrestrial electron 
superluminality are extremely tight. At LEP [57], synchrotron radiation measurements at $\mathrm{GeV}$ energies yield a maximum fractional superluminality $(v-c) / c$ of $5 \times 10^{-15}$. This implies that either the neutrino superluminality disappears before the electroweak scale, or else some conspiracy of other effects prevents it from being transmitted to the electron.

In [11] the authors noted, as was shown in $[42,43]$, that in the case of a coupling $v^{\dagger} \partial_{0} v$, a one-loop mixing generates the operator $e^{\dagger} \partial_{0} e$ which leads to a superluminality for electrons which is suppressed by at most $E^{2} / M_{W}^{2}$ with respect to that of neutrinos and so is 5 orders of magnitude above the LEP bound quoted above. More complicated breaking models, such as the coupling to sterile neutrinos [11, 42, 43] and supersymmetric completions [11], are able to push these effects onto higher derivative operators. However, generically this merely increases the exponent of the $E / M_{W}$ suppression.

One might hope that there exist flavor-asymmetric Lorentz-violating terms which evade the neutrino oscillation constraints from KamLAND. In this case one could simply declare that Lorentz-violation only occurs for muon and perhaps tau neutrinos, hoping that their mixing with electrons is sufficiently suppressed to avoid the LEP synchrotron bound. There are a number of reasons why this approach fails, but we have seen (Section 4.2) that our terms certainly do not allow such an evasion of the flavor neutrality constraints.

\section{Acknowledgments}

J. Evslin is supported by the Chinese Academy of Sciences Fellowship for Young International Scientists Grant no. 2010Y2JA01. E. Ciuffoli, J. Liu and X. Zhang are supported in part by the NSF of China.

\section{References}

[1] T. Adam, N. Agafonova, and A. Aleksandrov, "Measurement of the neutrino velocity with the OPERA detector in the CNGS beam," Journal of High Energy Physics, vol. 2012, article 93, 2012.

[2] A. Zichichi, "Results from LVD-OPERA Combined Analysis: A Time-Shift in the OPERA Setup," http://agenda.infn.it/getFile.py/access? resId=0\&materialId=slides $\&$ confId $=4896$.

[3] N. Y. Agafonova, P. Antonioli, V. V. Ashikhmin et al., "Determination of a time-shift in the OPERA set-up using high energy horizontal muons in the LVD and OPERA detectors," The European Physical Journal Plus, vol. 127, article 71, 2012.

[4] P. Adamson, "Neutrino velocity: results and prospects of experiments at other beamlines," Neutrino, 2012.

[5] P. A. Sanchez, R. Barzaghib, G. Bellini et al., "Measurement of CNGS muon neutrino speed with Borexino," Physics Letters B, vol. 716, pp. 401-405, 2012.

[6] F. Ronga, "Analysis of the MACRO experiment data to compare particles arrival times under Gran Sasso," In press, http://arxiv.org/abs/1208.0791.

[7] N. Y. Agafonova, M. Aglietta, P. Antonioli et al., "Measurement of the velocity of neutrinos from the CNGS beam with the Large Volume Detector," Physical Review Letters, vol. 109, Article ID 070801, 5 pages, 2012.
[8] M. Antonello, B. Baibussinov, P. Benetti et al. et al., "Precision measurement of the neutrino velocity with the ICARUS detector in the CNGS beam," Journal of High Energy Physics, vol. 2012, article 49, 2012.

[9] K. Hirata, T. Kajita, M. Koshiba et al., "Observation of a neutrino burst from the supernova SN 1987a," "Physical Review Lettersm, vol. 58, pp. 1490-1493, 1987.

[10] R. M. Bionta, G. Blewitt, C. B. Bratton et al., "Observation of a neutrino burst in coincidence with supernova 1987A in the large magellanic cloud," Physical Review Letters, vol. 58, no. 14, pp. 1494-1496, 1987.

[11] G. F. Giudice, S. Sibiryakov, and A. Strumia, "Interpreting OPERA results on superluminal neutrino," Nuclear Physics B, vol. 861, no. 1, pp. 1-16, 2012.

[12] A. G. Cohen and S. L. Glashow, "Pair creation constrains superluminal neutrino propagation," Physical Review Letters, vol. 107, Article ID 181803, 2011.

[13] X.-J. Bi, P.-F. Yin, Z.-H. Yu, and Q. Yuan, "Constraints and tests of the OPERA superluminal neutrinos," Physical Review Letters, vol. 107, Article ID 241802, 2011.

[14] G. Amelino-Camelia, L. Freidel, J. Kowalski-Glikman, and L. Smolin, "OPERA neutrinos and relativity," Modern Physics Letters A, vol. 27, no. 10, Article ID 125006, 2012.

[15] Y. Ling, "A note on superluminal neutrinos and deformed special relativity," In press, http://arxiv.org/abs/1111.3716.

[16] G. Dvali and A. Vikman, "Price for environmental neutrinosuperluminality," Journal of High Energy Physics, vol. 2012, article 134, 2012.

[17] L. Iorio, "Constraints from orbital motions around the Earth of the environmental fifth-force hypothesis for the OPERA superluminal neutrino phenomenology," Journal of High Energy Physics, vol. 2012, article 73, 2012.

[18] J. Evslin, "Challenges confronting superluminal neutrino models," International Journal of Modern Physics: Conference Series, vol. 10, pp. 159-168.

[19] A. Hebecker and A. Knochel, "The price of neutrino superluminality continues to rise," Physics Letters B, vol. 715, no. 1-3, pp. 116-120, 2012.

[20] P. Gu, X. Wang, and X. Zhang, "Dark energy and neutrino mass limits from baryogenesis," Physical Review D, vol. 68, no. 8, Article ID 087301, 2003.

[21] R. Fardon, A. E. Nelson, and N. Weiner, "Dark energy from mass varying neutrinos," Journal of Cosmology and Astroparticle Physics, vol. 2004, Article ID 005, 2004.

[22] A. Aguilar-Arevalo, L. B. Auerbach, R. L. Burman et al., "Evidence for neutrino oscillations from the observation of $\bar{v}_{e}$ appearance in a $\bar{v}_{\mu}$ beam," Physical Review D, vol. 64, Article ID 112007, 22 pages, 2001.

[23] A. A. Aguilar-Arevalo, A. O. Bazarko, S. J. Brice et al., "A Search for electron neutrino appearance at the $\Delta m^{2} \sim 1 \mathrm{eV}^{2}$ scale," Physical Review Letters, vol. 98, Article ID 231801, 2007.

[24] A. A. Aguilar-Arevalo, C. E. Anderson, A. O. Bazarko et al., "Unexplained excess of electron-like events from a $1-\mathrm{GeV}$ neutrino beam," Physical Review Letters, vol. 102, Article ID 101802, 2009.

[25] A. A. Aguilar-Arevalo, C. E. Anderson, S. J. Brice et al., "Event excess in the MiniBooNE search for $\bar{v}_{\mu} \rightarrow \bar{v}_{e}$ oscillations," Physical Review Letters, vol. 105, no. 18, Article ID 181801, 2010.

[26] A. A. Aguilar-Arevalo, B. C. Brown, L. Bugel, G. Cheng et al., "A combined $v_{\mu} \rightarrow v_{e}$ and $\bar{v}_{\mu} \rightarrow \bar{v}_{e}$ oscillation analysis of the MiniBooNE excesses," In press, http://arxiv.org/abs/1207.4809. 
[27] B. Armbruster, I. M. Blair, B. A. Bodmann et al., "Upper limits for neutrino oscillations $\bar{v}_{\mu} \rightarrow \bar{v}_{e}$ from muon decay at rest," Physical Review D, vol. 65, no. 11, Article ID 112001, 16 pages, 2002.

[28] M. Antonello, B. Baibussinov, P. Benetti et al. et al., "Experimental search for the LSND anomaly with the ICARUS LAr TPC detector in the CNGS beam," . In press, http://arxiv.org/abs/1209.0122.

[29] P. Anselmann, R. Fockenbrock, W. Hampel et al., "First results from the ${ }^{51} \mathrm{Cr}$ neutrino source experiment with the GALLEX detector," Physics Letters B, vol. 342, pp. 440-450, 1995.

[30] W. Hampela, G. Heussera, J. Kiko et al., "Final results of the ${ }^{51} \mathrm{Cr}$ neutrino source experiments in GALLEX," Physics Letters B, vol. 420, no. 1-2, pp. 114-126, 1998.

[31] J. N. Abdurashitov, V. N. Gavrin, S. V. Ginn et al., "The Russian-American Gallium Experiment (SAGE) Cr neutrino source measurement," Physical Review Letters, vol. 77, no. 23, pp. 4708-4711, 1996.

[32] J. N. Abdurashitov, V. N. Gavrin, S. V. Girin et al., "Measurement of the response of the Russian-American gallium experiment to neutrinos from a ${ }^{51} \mathrm{Cr}$ source," Physical Review C, vol. 59, pp. 2246-2263, 1999.

[33] J. N. Abdurashitov, V. N. Gavrin, S. V. Girin et al., "Measurement of the response of a Ga solar neutrino experiment to neutrinos from a ${ }^{37}$ Ar source," Physical Review C, vol. 73, no. 4, Article ID 045805, 2006.

[34] J. N. Abdurashitov, V. N. Gavrin, V. V. Gorbachev et al., "Measurement of the solar neutrino capture rate with gallium metal. III: results for the 2002-2007 data-taking period," Physical Review C, vol. 80, no. 1, Article ID 015807, 16 pages, 2009.

[35] G. Mention, M. Fechner, T. Lasserre et al., "Reactor antineutrino anomaly," Physical Review D, vol. 83, no. 7, Article ID 073006, 2011.

[36] E. Ciuffoli, J. Evslin, and H. Li, "The reactor anomaly after daya bay and RENO," In press, http://arxiv.org/abs/1205.5499.

[37] P. C. de Holanda and A. Y. Smirnov, "Homestake result, sterile neutrinos and lowenergy solar neutrino experiments," Physical Review D, vol. 69, Article ID 113002, 8 pages, 2004.

[38] P. C. De Holanda and A. Y. Smirnov, "Solar neutrino spectrum, sterile neutrinos, and additional radiation in the Universe," Physical Review D, vol. 83, no. 11, Article ID 113011, 2011.

[39] R. Keisler, C. L. Reichardt, K. A. Aird et al. et al., "A measurement of the damping tail of the cosmic microwave background power spectrum with the south pole telescope," The Astrophysical Journal, vol. 743, p. 28, 2011.

[40] E. Komatsu, K. M. Smith, J. Dunkley et al., "Seven-year wilkinson microwave anisotropy probe (WMAP) observations: cosmological interpretation," The Astrophysical Journal Supplement Series, vol. 192, no. 2, Article ID 18, 2011.

[41] R. Abbasi, Y. Abdou, T. Abu-Zayyad et al., "Measurement of the atmospheric neutrino energy spectrum from $100 \mathrm{GeV}$ to 400 TeV with IceCube," Physical Review D, vol. 83, no. 1, Article ID 012001, 2011.

[42] P.-H. Gu, X.-J. Bi, and X.-M. Zhang, "Dark energy and neutrino CPT violation," The European Physical Journal C, vol. 50, pp. 655-659, 2007.

[43] S. Ando, M. Kamionkowski, and I. Mocioiu, "Neutrino oscillations, Lorentz/CPT violation, and dark energy," Physical Review $D$, vol. 80, no. 12, Article ID 123522, 2009.

[44] J. Alexandre, J. Ellis, and N. E. Mavromatos, "On the possibility of superluminal neutrino propagation," Physics Letters B, vol. 706, no. 4-5, pp. 456-461, 2012.
[45] A. Kehagias, "Relativistic superluminal neutrinos," . In press, http://arxiv.org/abs/1109.6312.

[46] T. Li and D. V. Nanopoulos, "Background dependent lorentz violation from string theory," In press, http://arxiv.org/abs/ 1110.0451 .

[47] A. Nicolis, R. Rattazzi, and E. Trincherini, "The Galileon as a local modification of gravity," Physical Review D, vol. 79, Article ID 064036, 2009.

[48] A. Nicolis and R. Rattazzi, "Classical and quantum consistency of the DGP model," Journal of High Energy Physics, vol. 2004, Article ID 059, 2004.

[49] A. I. Vainshtein, "To the problem of nonvanishing gravitation mass," Physics Letters B, vol. 39, no. 3, pp. 393-394, 1972.

[50] J. Evslin and T. Qiu, "Closed timelike curves in the galileon model," Journal of High Energy Physics, vol. 11, Article ID 032, 2011.

[51] S. Abe, T. Ebihara, S. Enomoto et al., "Precision measurement of neutrino oscillation parameters with KamLAND," Physical Review Letters, vol. 100, Article ID 221803, 5 pages, 2008.

[52] L. Wolfenstein, "Neutrino oscillations in matter," Physical Review D, vol. 17, pp. 2369-2374, 1978.

[53] S. P. Mikheev and A. Y. Smirnov, "Resonance amplification of oscillations in matter and spectroscopy of solar neutrinos," Soviet Journal of Nuclear Physics, vol. 42, pp. 913-917, 1985.

[54] S. P. Mikheev and A. Y. Smirnov, "Resonance enhancement of oscillations in matter and solar neutrino spectroscopy," Yadernaya Fizika, vol. 42, pp. 1441-1448, 1985.

[55] A. Strumia and F. Vissani, "Neutrino masses and mixings and...," In press, http://arxiv.org/abs/hepph/0606054.

[56] K. Nomoto, "Evolution of 8 to 10 solar mass stars toward electron capture supernovae II," Astrophysical Journal, vol. 322, p. 206, 1987.

[57] B. Altschul, "Bounding isotropic Lorentz violation using synchrotron losses at LEP," Physical Review D, vol. 80, no. 9, Article ID 091901, 2009. 

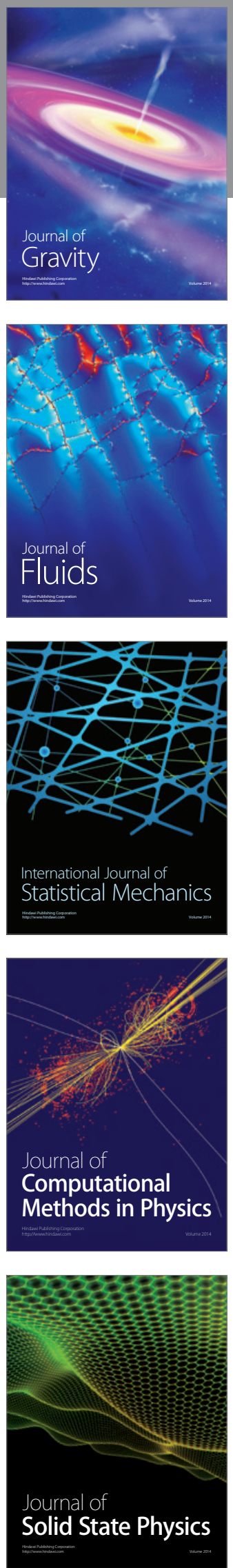

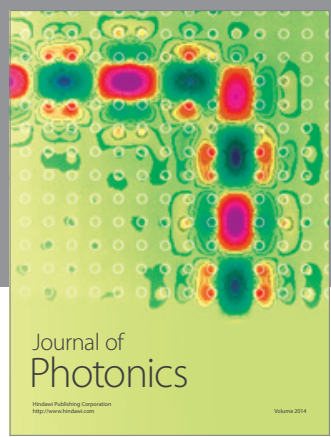

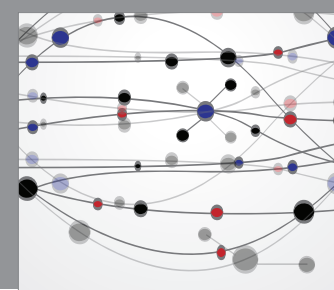

The Scientific World Journal

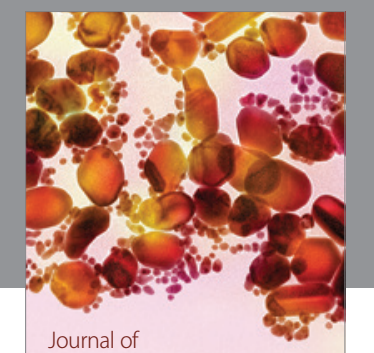

Soft Matter
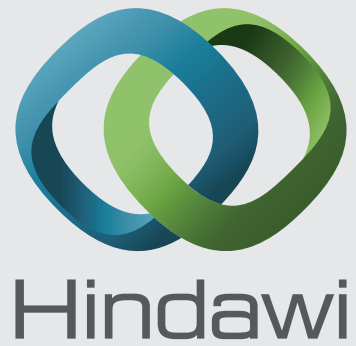

Submit your manuscripts at

http://www.hindawi.com
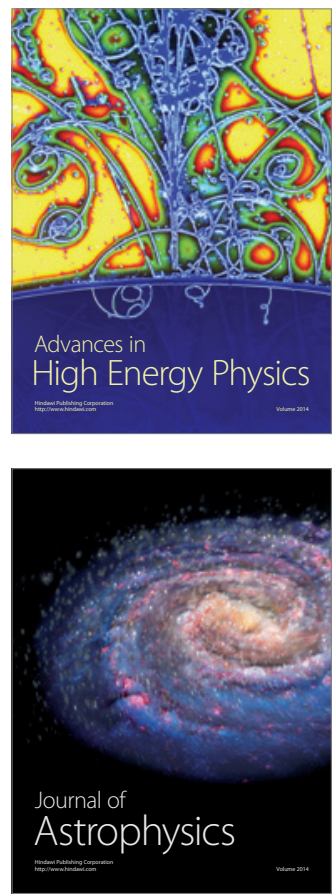
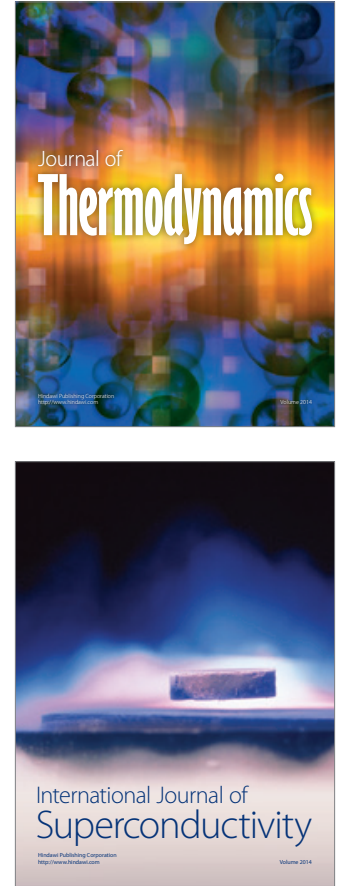
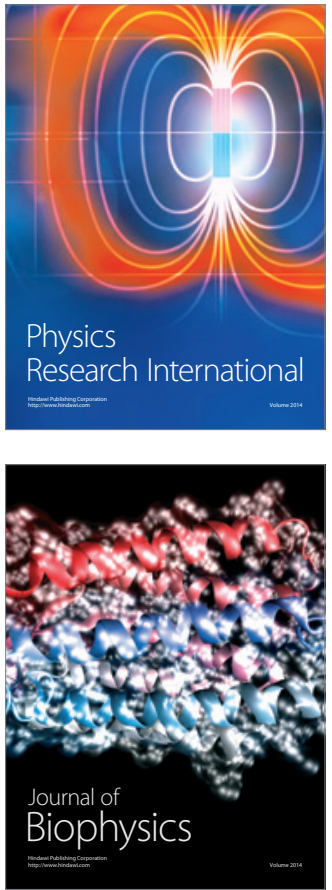
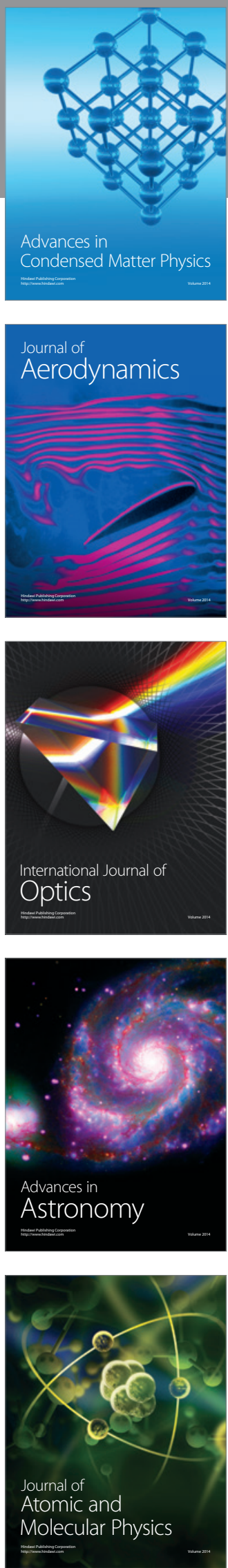